\title{
Análise Estatística e Geoestatística da Precipitação Média para o Município de Joinville (SC)
}

\author{
Yara Rúbia de Mello ${ }^{1}$, Therezinha Maria Novais de Oliveira ${ }^{2}$ \\ ${ }^{1}$ Programa de Pós-Graduação em Geografia, Universidade Federal do Paraná, \\ Curitiba, PR, Brasil. \\ ${ }^{2}$ Programa de Pós-Graduação em Saúde e Meio Ambiente, \\ Universidade da Região de Joinville, Joinville, SC, Brasil.
}

Received: 13/4/2015 - Accepted: 26/8/2015

\begin{abstract}
Resumo
A precipitação pluviométrica é um fator importante na definição do clima de uma localidade, sendo o resultado do conjunto de eventos meteorológicos e geográficos. Seu estudo é necessário devido à forte influência que exerce sobre as condições ambientais e socioeconômicas. Assim, faz-se necessário a realização de estudos de distribuição pluviométrica que contribuam para uma melhor gestão municipal socioambiental. Portanto, neste estudo, foram utilizados dados pluviométricos de 42 pluviômetros na região de Joinville (SC) objetivando mapear a precipitação média anual e mensal do município. Primeiramente foi realizada análise exploratória dos dados seguido do teste de homogeneidade, na sequência realizou-se análise da dependência espacial dos dados através do semivariograma esférico, exponencial e gaussiano. Por fim, foram geradas as superfícies interpoladas de precipitação pelo método de krigagem e cokrigagem. Para selecionar a melhor superfície foi utilizada à validação cruzada e a análise do IDE. O mapeamento foi realizado utilizando o software ArcGIS. Os resultados mostraram que, o modelo esférico na krigagem e gaussiano na cokrigagem, apresentam bom desempenho, porém a krigagem foi o método que apresentou melhores resultados em todos os parâmetros de validação, gerando uma média anual de precipitação de 2.130,1 mm e mensal de 183,6 mm para Joinville.
\end{abstract}

Palavras-chave: semivariograma, krigagem, cokrigagem.

\section{Statistical and Geostatistical Analysis of the Average Rainfall in the Municipality of Joinville (SC)}

\begin{abstract}
Rainfall is an important factor in climate definition of a locality, being the result of the whole of meteorological and geographic events. Its study is necessary due to the strong influence it exerts upon the environmental and socioeconomic conditions. Thus, is necessary to undertake studies of rainfall distribution contributing to a better socio-environmental municipal administration. Therefore, rainfall data on 42 samples of the Joinville (SC) area were used in this study, seeking to map the municipality's average yearly and monthly rainfall. At first, an exploratory analysis of the data was undertaken, followed by the homogeneity test. Afterwards, an analysis of the spatial dependence of the data through the spherical, exponential and gaussian semivariograms was conducted. Finally, the interspersed rainfall surfaces were generated by the kriging and cokriging method. To select the best surface, cross-validation and SDI analysis were used. The mapping was performed with the ArcGIS software. The results show that the spherical template, in the kriging, and the gaussian, in the cokriging, show good performance, but the kriging was the method that showed better results in validation parameters, generating an yearly average rainfall of 2,130.1 $\mathrm{mm}$, and a monthly average rainfall of $183.6 \mathrm{~mm}$ for Joinville.
\end{abstract}

Keywords: Semivariogram, kriging, cokriging.

Autor de correspondência: Yara Rúbia de Mello, yarademello@gmail.com. 


\section{Introdução}

A precipitação pluviométrica é uma variável climática que possui uma distribuição aleatória no espaço e no tempo, exercendo forte influência nas condições ambientais. Os equipamentos comumente utilizados para medir a quantidade de chuva que cai sobre uma região são chamados de pluviômetros e pluviógrafos (automáticos), existindo um conjunto destes, forma-se uma rede que possibilita o estudo climatológico de uma determinada área seja uma bacia hidrográfica ou o limite administrativo de um município (Tucci, 2001; Moulin, 2005; Caram, 2007).

A série temporal de dados de chuva geradas pelos pluviômetros e pluviógrafos será homogênea se as variações forem causadas pelo tempo e clima, uma vez que a homogeneidade é representada por uma série de dados climáticos que tenham variações causadas por características físicas. Porém, não é uma tarefa simples ao pesquisador determinar a homogeneidade da série, o acesso ao metadado é um instrumento que possibilita verificar se o equipamento foi transferido de local, as mudanças na paisagem e manutenção. Sendo um exemplo de dificuldade na análise, pois os fatores não climáticos interferem na homogeneidade, gerando uma tendência gradual dos dados e/ou descontinuidade na variância (Tucci, 2001; Caram, 2007).

Quando se realiza o mapeamento de uma variável climática a partir de estações de superfície tem-se a problemática de generalizar o resultado para uma área a partir de amostras pontuais. A precipitação apresenta certo grau de dependência espacial, desta forma a geoestatística ou teoria das variáveis regionalizadas é uma alternativa na análise amostral, pois permite definir o raio de dependência entre os exemplares. No conceito fundamental da geoestatística tem-se que as amostras mais próximas no tempo e no espaço sejam mais similares entre si (Baú et al., 2006; Caram, 2007).

O semivariograma é uma função matemática fundamental na aplicação da geoestatística, ele determina, sobretudo a dependência entre duas variáveis aleatórias regionalizadas locais, levando em consideração à auto correlação em função da distância e da direção delas. É pré-requisito para os métodos de interpolação de krigagem e cokrigagem, se houver dependência espacial entre as amostras podem-se estimar valores para os locais não amostrados (Carvalho et al., 2004; Mello, 2004; Mello et al., 2005; Moulin, 2005; Baú et al., 2006).

Krigagem é um método univariado de inferência espacial que usa a dependência no espaço expressa no semivariograma entre amostras vizinhas para estimar valores em qualquer posição da área de estudo, sem tendência e com variância mínima, tornando-se um ótimo estimador. A utilização da krigagem possibilita o cálculo de uma medida do erro de estimação para cada valor e de um intervalo de confiança (Mello, 2004; Moulin, 2005; Baú et al., 2006; Carvalho et al., 2012). Este método vem sendo empregado em diversos estudos como em Mello et al. (2003), Carvalho et al. (2005), Moulin (2005), Caram (2007), Bargaoui et al. (2009), Viola et al. (2010), Lellis et al. (2011) e Wanderley et al. (2012).

A cokrigagem é um método multivariado com mais de uma variável utilizada na análise da amostra. Geralmente nos estudos de distribuição pluviométrica utiliza-se a variável altitude devido a forte influência que o relevo exerce sobre o clima. Justamente nas áreas montanhosas é que se tem uma maior escassez de equipamentos que medem a pluviosidade, interferindo na qualidade dos mapeamentos (Carvalho et al., 2002; Diodato et al., 2005; Viola et al., 2010). Este método apesar de ser utilizado com menor frequência que a krigagem, já foi descrito em muitos estudos, como em Hevesi et al. (1992), Carvalho et al. (2002), Diadato et al. (2005), Viola et al. (2010) e Carvalho et al. (2012).

O objetivo desta pesquisa foi realizar um mapeamento pluviométrico para Joinville (SC), município que possui uma média anual de precipitação de $2.200 \mathrm{~mm}$. A distribuição pluviométrica regional é influenciada pela presença marcante da serra (altitudes superiores a mil metros) em conjunto a proximidade do Oceano Atlântico, e aos sistemas atmosféricos atuantes, com destaque para os sistemas frontais durante todo o ano (Rodrigues et al., 2004; Oliveira, 2006; Mello et al., 2013).

\section{Material e Métodos}

\section{1. Área de estudo}

O município de Joinville está localizado na região nordeste do Estado de Santa Catarina, conforme Fig. 1, neste mapa é possível observar a presença marcante das montanhas na configuração do relevo, sendo o Pico Joinville $(1.350 \mathrm{~m})$ o ponto culminante. Na compartimentação topográfica municipal também se destaca a presença do planalto e da planície (Oliveira, 2006).

A zona urbana de Joinville está localizada na planície costeira, onde os índices pluviométricos são inferiores aos encontrados próximos a escarpa da serra, e superiores aos da região de planalto (Mello et al., 2013). As regiões de planalto e serra estão inseridas predominantemente na área rural, por isso, a taxa populacional é menor que a urbana (Ippuj, 2013), na região da Serra Queimada, Serra do Quiriri e Serra do Piraí se encontra importantes mananciais que abastecem o município.

Devido aos altos índices pluviométricos, ambas as porções do relevo são atingidas por desastres naturais como inundações e movimentos de massa. A alta pluviosidade aliada à influência da maré alaga comumente a região central da cidade as margens dos rios da bacia hidrográfica do rio Cachoeira. Isto ocorre principalmente nos meses de verão, época mais chuvosa, apresentando uma média de $240,9 \mathrm{~mm}$ de chuva, podendo atingir mais de $600 \mathrm{~mm}$ no mês de janeiro, segundo dados da Estação Meteorológica da Univille (Mello et al., 2013). 


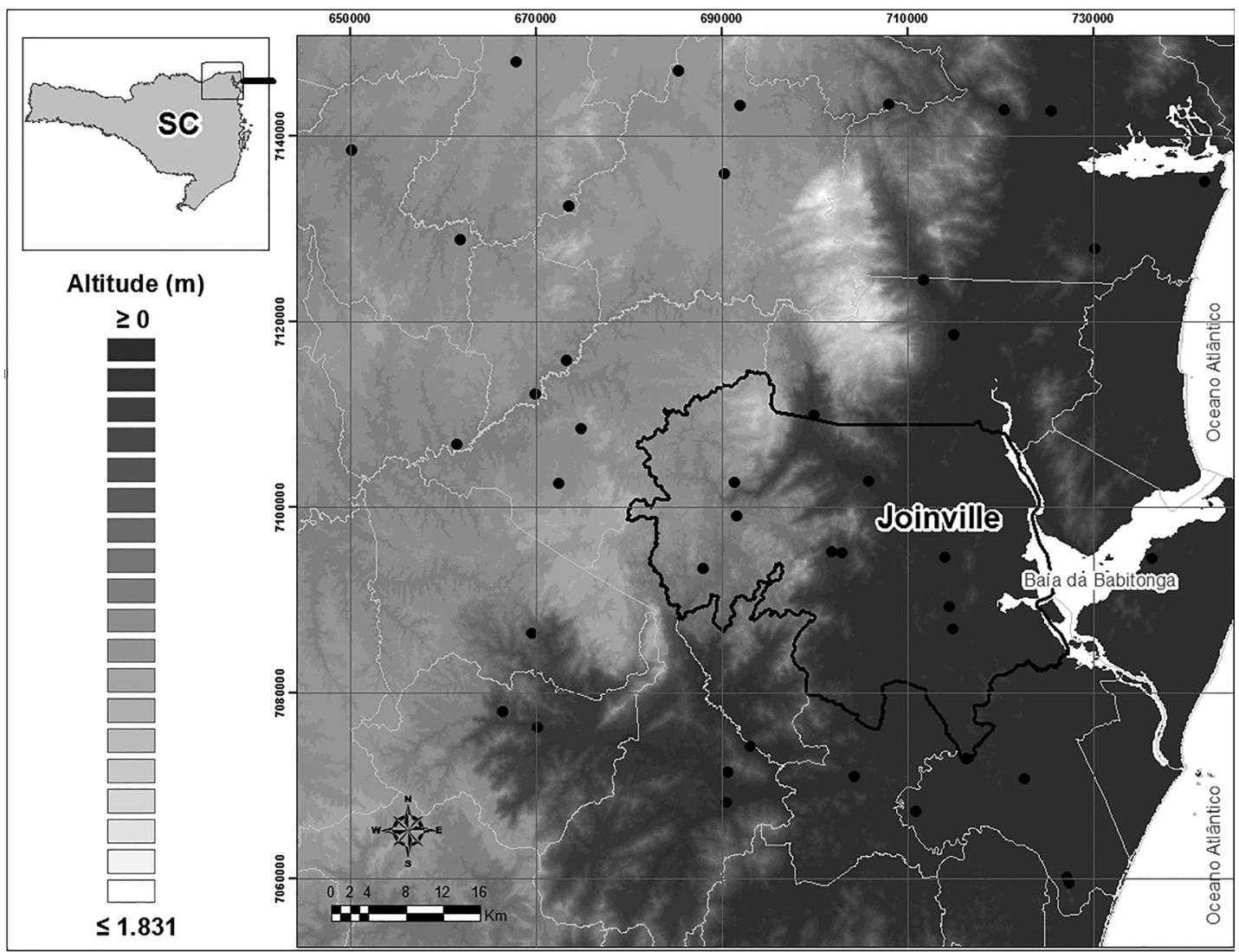

Figura 1 - Localização da área de estudo e distribuição da rede de pluviômetros. Org.: Yara de Mello, 2014.

\subsection{Rede de pluviômetros}

Foram utilizados dados de 42 postos pluviométricos localizados na região de Joinville (Fig. 1) e utilizados no trabalho de Mello et al. (2013) para caracterizar a distribuição pluviométrica na região nordeste do Estado de Santa Catarina. Neste contexto, os dados compreendem a média anual de 55 anos (1953 a 2008) e mensal de 10 anos (1980 a 1989).

\subsection{Análise dos dados pluviométricos}

A análise exploratória dos dados possibilita a identificação de valores discrepantes e a forma da distribuição da variável. Realizou-se a distribuição de frequência utilizando-se o cálculo $5\left[\log _{10}(\mathrm{~N})\right]$ para determinar o número de classes, o intervalo de classes foi definido por [Valor máximo - (Valor mínimo - 1)]/Número de classes. A partir disso foram analisadas medidas de tendência e de dispersão da amostra (Vieira, 1980; Assis, 1996). As ferramentas de análise exploratória não permitem descrever a variabilidade espacial dos dados, pois elas não incorporam a informação espacial.

Posteriormente com o intuito de testar a homogeneidade das variáveis fez-se a análise de consistência através do Método de Dupla Massa descrito por Tucci (2001). Este procedimento consiste em selecionar os postos de uma região e plotar num gráfico cartesiano os valores acu- mulados do posto de referência em relação à média acumulada dos postos da região. Haverá consistência quando houver uma tendência linear entre as análises, sendo avaliada pelo ajuste da equação da reta e pelo Coeficiente de Determinação $\left(r^{2}\right)$.

Desta forma foi utilizada a divisão por localidade geográfica dos pluviômetros, sendo elas A) planície costeira; B) proximidades da frente da escarpa da Serra do Mar; C) escarpa da Serra do Mar e D) planalto. Foi selecionado um pluviômetro de referência, que possuía a maior série de dados sem falhas por compartimentação topográfica, para ser comparado aos outros pluviômetros da localidade, formando o grupo da região. A divisão dos grupos por compartimentação topográfica esta descrita em Mello et al. (2013). Toda a organização dos dados, cálculos e gráficos foram efetuados no Microsoft Excel 2010, Statistica 8 e Minitab 17.

\subsection{Semivariograma}

Para a utilização do método de krigagem e cokrigagem o semivariograma ou variograma é a ferramenta básica, pois permite analisar quantitativamente a variação de um fenômeno regionalizado. As propriedades típicas de um variograma analisadas foram: alcance (a), efeito pepita $\left(\mathrm{C}_{\mathrm{O}}\right)$, variância estrutural $(\mathrm{C})$ e patamar $\left(\mathrm{C}_{\mathrm{O}}+\mathrm{C}\right)$. Foram utilizados nesta pesquisa os modelos teóricos de vario- 
gramas (com patamar) esférico, exponencial e gaussiano. Através da análise do semivariograma é possível perceber se o fenômeno é isotrópico (a função variograma não muda com a direção) ou anisotrópico (a função variograma se altera com a direção), desta forma a covariância foi calculada em quatro direções horizontais: $0^{\circ}, 45^{\circ}, 90^{\circ}$ e $135^{\circ}$ (Andriotti, 2003; Carvalho et al., 2004; Viola et al., 2010; Lellis et al., 2011; Wanderley et al., 2012; Yamamoto et al., 2013).

Foi verificado o Índice de Dependência Espacial (IDE), descrito por Cambardella et al. (1994) apud Caram (2007). Este índice determina faixas de dependência espacial entre a amostra e permite calcular o grau de dependência em função da relação entre o efeito pepita $(\mathrm{Co})$ e o patamar $(\mathrm{Co}+\mathrm{C})$. O IDE é descrito pela equação:

$$
\mathrm{IDE}=\frac{\mathrm{Co}}{\mathrm{Co}+\mathrm{C}} \times 100
$$

IDE $\leq 25 \%$ - forte dependência espacial; IDE entre $25 \%$ e $75 \%$ - moderada dependência espacial; IDE $\geq 75 \%$ - fraca dependência espacial; IDE $=100 \%$ - variável independente espacialmente (efeito pepita puro).

A análise do semivariograma foi realizada no software ArcMap 10.2.2 através da extensão Geostatistical Analyst.

\subsection{Krigagem e cokrigagem}

Após verificar a dependência espacial da amostra através do semivariograma é possível estimar valores de precipitação para os locais não amostrados dentro da área de análise através da técnica de krigagem. Estimativas geoestatísticas são muitas vezes superiores aos demais métodos de interpolação justamente pela utilização da função variograma, que depende da existência ou não do efeito pepita, da amplitude e da presença de anisotropia. (Alvares, 2011; Wanderley et al., 2012; Yamamoto et al., 2013).

Foi selecionada para esta pesquisa a krigagem ordinária, por ser o método mais utilizado devido a sua simplicidade e os resultados que proporciona. Utilizaram-se os valores de erro (diferença entre valor medido e previsto) para gerar o mapa de resíduos pelo método do Inverso do Quadrado da Distância (IQD) (Andriotti, 2003; Yamamoto et al., 2013).

A cokrigagem é uma técnica pela qual se pode estimar diversas variáveis regionalizadas em conjunto, baseada na correlação espacial entre si. É fundamental para sua utilização a verificação da correlação existente entre a variável primária e as variáveis secundárias (Yamamoto et al., 2013).

Neste trabalho foi utilizado a cokrigagem ordinária com os dados de precipitação e os dados de altitude das 42 estações e de mais 30 amostras inseridas na área de estudo e selecionadas as altitudes através do raster de elevação do Shuttle Radar Topography Mission (SRTM) para dar a heterotopia parcial ao conjunto de dados.
Toda a análise foi realizada no ArcMap 10.2.2 e a técnica de validação cruzada foi utilizada para definir o melhor ajuste de modelo. Os mapas de krigagem, cokrigagem e IQD foram elaborados com base digital do Instituto Brasileiro de Geografia e Estatística (IBGE), Comitê Cubatão Cachoeira (CCJ) e raster SRTM da Nasa, utilizando a projeção UTM (zona 22 sul) e Datum Sirgas 2000, a escala numérica utilizada foi 1: 250.000 .

\section{Resultados e Discussão}

\subsection{Análise exploratória e homogeneidade dos dados anuais e mensais}

Foi selecionada para o mapeamento pluviométrico da região de Joinville uma amostra (Fig. 1) não probabilística de dados quantitativos contínuos, referente a um estudo observacional e retrospectivo (Triola, 2008).

Na Tabela 1 é possível observar a distribuição de frequência para os dados anuais, foram utilizadas 8 classes, definidos os pontos médios e as frequências $(f)$, frequências acumuladas $(f a)$, frequências relativas $(f r)$ e frequências relativas acumuladas (fra).

Na Fig. 2 é possível observar o histograma de frequência (A), o polígono de frequência acumulada ou ogiva (B) e o boxplot (C), utilizados como apoio na visualização dos dados. Relacionando a distribuição de frequência (Tabela 1) com os gráficos de distribuição (Fig. 2), é possível notar que a maior frequência de dados se encontra no ponto médio de $1.957 \mathrm{~mm}$ de chuva, ou seja, variando entre $1.843,8 \mathrm{~mm}$ e $2.070,8 \mathrm{~mm}$. A maior parte da amostra, $90 \%$, apresenta média inferior a $2.524,8 \mathrm{~mm}$, ou seja, apenas 4 elementos possuem média superior a este valor, e todos estão localizados nas proximidades da frente da escarpa da Serra do Mar. A Fig. 2 - C aponta a configuração de um dado discrepante ou outlier, correspondente ao valor de 3.204,4 mm, ou seja, a amostra é simétrica e proveniente de uma distribuição normal.

As medidas de tendência central para o grupo anual foram: média aritmética (1.945), média aritmética dos dados agrupados (1.984), mediana (1.875) e mediana dos da-

Tabela 1 - Distribuição de frequência dos totais anuais de chuva da região de Joinville (SC).

\begin{tabular}{lccccc}
\hline Classe & P. médio $(\mathrm{X})$ & $f$ & $f \mathrm{a}$ & $f \mathrm{r}$ & $f \mathrm{ra}$ \\
\hline $1389,8-1616,8$ & 1503 & 10 & 10 & 24 & 24 \\
$1616,8-1843,8$ & 1730 & 5 & 15 & 12 & 36 \\
$1843,8-2070,8$ & 1957 & 13 & 28 & 31 & 67 \\
$2070,8-2297,8$ & 2184 & 5 & 33 & 12 & 79 \\
$2297,8-2524,8$ & 2411 & 5 & 38 & 12 & 90 \\
$2524,8-2751,8$ & 2638 & 2 & 40 & 5 & 95 \\
$2751,8-2978,8$ & 2865 & 1 & 41 & 2 & 98 \\
$2978,8-3205,8$ & 3092 & 1 & 42 & 2 & 100 \\
Totais & & 42 & & 100 & 100 \\
\hline
\end{tabular}



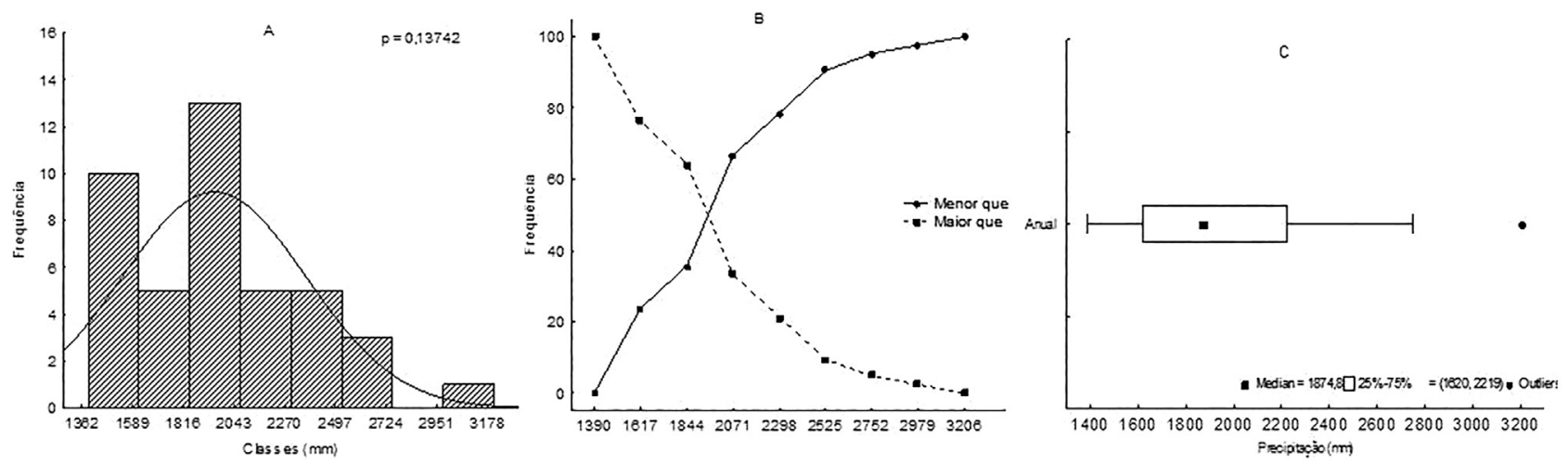

Figura 2 - Gráficos de distribuição dos dados anuais: histograma de frequência (A); polígono de frequência acumulada ou ogiva, indicando os eventos menores ou iguais a determinada frequência na forma ascendente e os eventos maiores ou iguais a determinada frequência na forma descendente (B) e boxplot $(\mathrm{C})$.

dos agrupados (1.949). Para os dados agrupados a média e mediana ficaram mais próximas, mas em ambos os casos, a média, por ser maior que a mediana, indicou leve assimetria positiva.

As medidas de dispersão calculadas foram amplitude (1.815 mm), variância (170255), desvio padrão (413) e

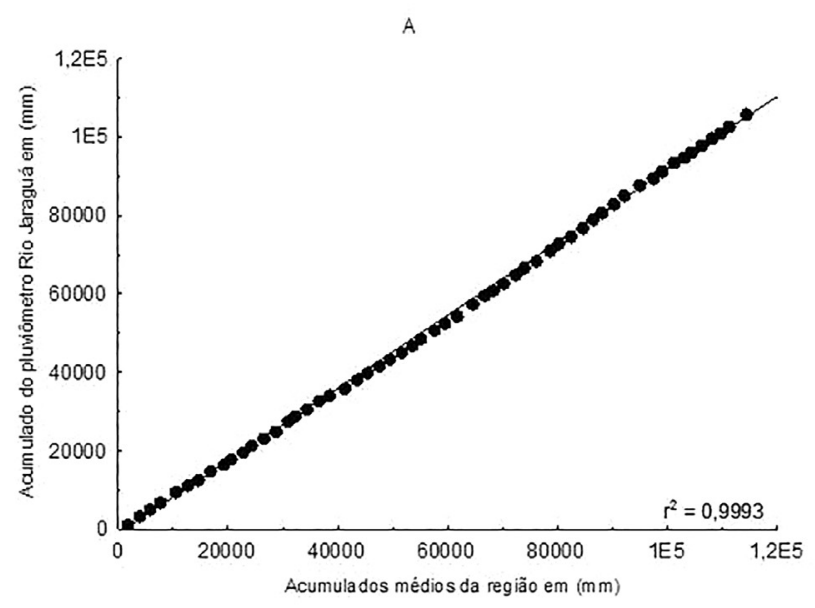

C

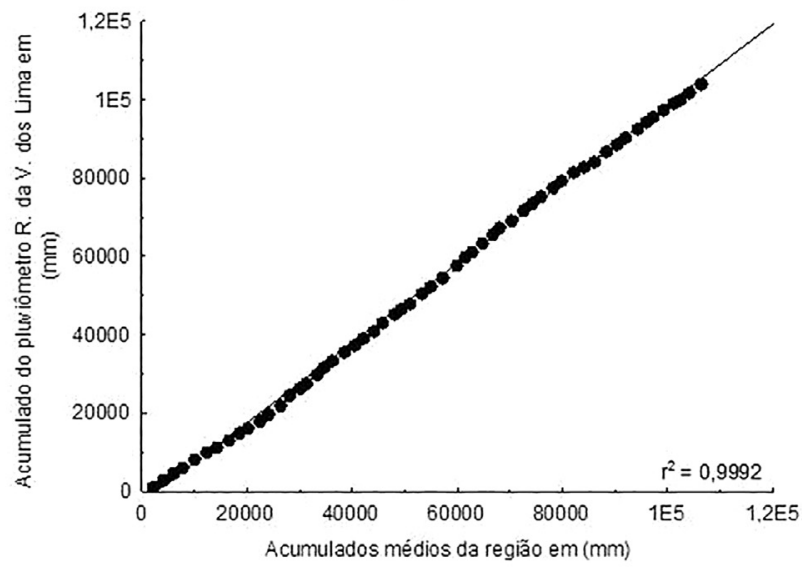

coeficiente de variação $21 \%$. A homogeneidade dos dados foi testada através do método de Dupla Massa, que apresentou para os coeficientes de determinação $\left(r^{2}\right)$ ótimos resultados, conforme Fig. 3, onde estão representados os gráficos de regressão relacionando os dados de chuvas da estação de referência para cada compartimentação topo-

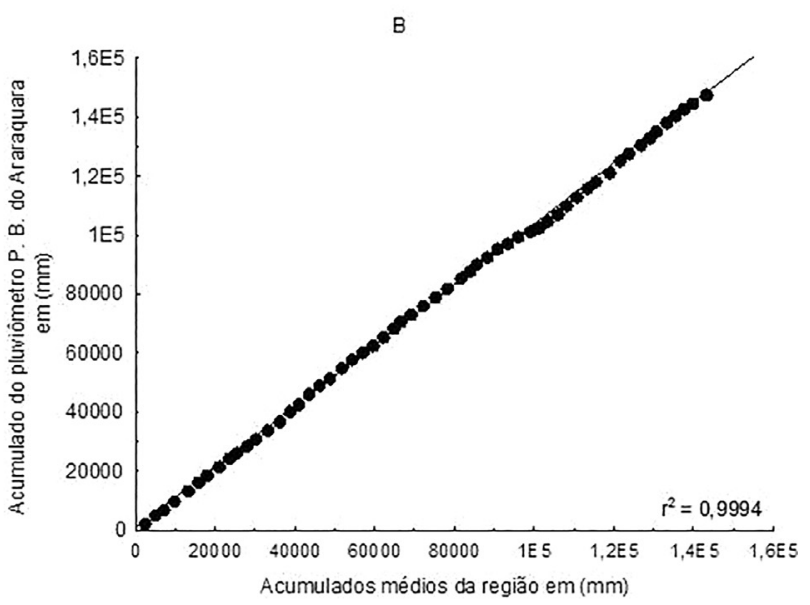

D

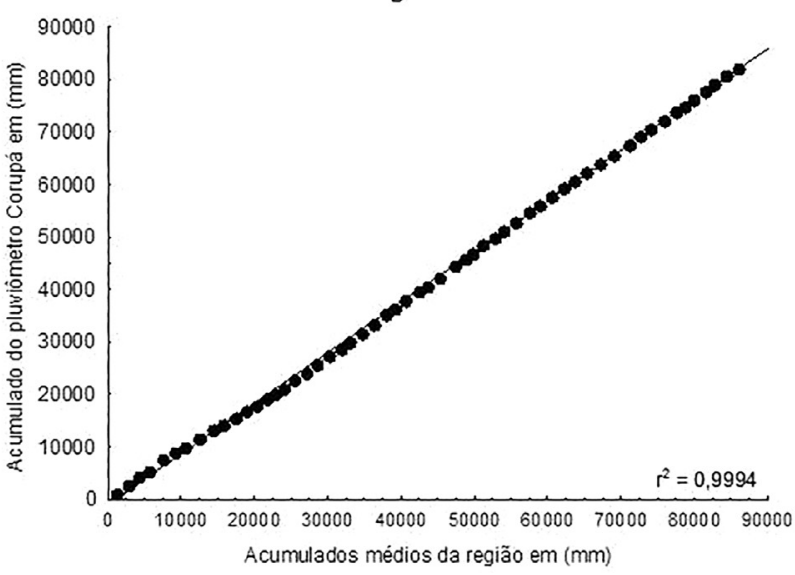

Figura 3 - Regressões lineares dos dados anuais acumulados de precipitação por localidade geográfica dos pluviômetros da planície costeira (A); proximidades da frente da escarpa da Serra do Mar (B); escarpa da Serra do Mar (C) e planalto (D). 
gráfica (variável dependente) e os outros postos da região (variável independente).

A Tabela 2 apresenta a distribuição de frequência para os dados mensais, observa-se uma concentração de dados entre a segunda e a quarta classe, ou seja, entre $122,3 \mathrm{~mm}$ e $194,3 \mathrm{~mm}$ de chuva.

Através da Fig. 4-A, B, C e da tabela 2 acima nota-se o mesmo padrão dos dados anuais, com $90 \%$ da amostra localizada até a quinta classe, média mensal máxima de $218,3 \mathrm{~mm}$ e as estações com maiores índices pluviométricos foram às mesmas, localizadas nas proximidades da serra. A análise de frequência é importante para se obter uma noção da distribuição dos valores da amostra e de sua localização no espaço, reafirmando a diferença na distribuição dos pluviômetros, as regiões montanhosas possuem maior escassez de equipamentos, porém maiores registros de chuva.

As medidas de tendência central calculadas para o grupo mensal foram média aritmética (168), média aritmética dos dados agrupados (169), mediana (166), mediana dos dados agrupados (164). Através da Fig. 4 -C é possível observar a concentração de dados abaixo dos $197 \mathrm{~mm}$ $(75 \%)$ e a mediana localizada abaixo deste valor, não houve configuração de outlier e o limite superior está consideravelmente mais distante da mediana que o limite inferior em relação às classes.

As medidas de dispersão encontradas foram amplitude (185), variância (1633), desvio padrão (40) e coeficiente de variação de $24 \%$. A homogeneidade dos dados mensais foi testada com os dados de chuva acumulados e correlacionados em gráficos de regressão linear, a partir do agrupamento segundo a compartimentação topográfica da localização dos pluviômetros (Fig. 5). Foram encontrados valores estatísticos significativos, semelhantes aos dados anuais.

\subsection{Análise dos semivariogramas}

Foram testados os semivariogramas para diferentes modelos teóricos, o melhor resultado das superfícies anuais e mensais interpoladas por krigagem se deu com o modelo linear esférico. Os erros foram comparados através da técnica de validação cruzada, resultado semelhante foi encon-
Tabela 2 - Distribuição de frequência das médias mensais de chuva da região de Joinville (SC).

\begin{tabular}{lccccc}
\hline Classe & P. médio $(\mathrm{X})$ & $f$ & $f \mathrm{a}$ & $f \mathrm{r}$ & fra \\
\hline $98,3-122,3$ & 110 & 4 & 4 & 10 & 10 \\
$122,3-146,3$ & 134 & 11 & 15 & 26 & 36 \\
$146,3-170,3$ & 158 & 8 & 23 & 19 & 55 \\
$170,3-194,3$ & 182 & 8 & 31 & 19 & 74 \\
$194,3-218,3$ & 206 & 7 & 38 & 17 & 90 \\
$218,3-242,3$ & 230 & 2 & 40 & 5 & 95 \\
$242,3-266,3$ & 254 & 1 & 41 & 2 & 98 \\
$266,3-290,3$ & 278 & 1 & 42 & 2 & 100 \\
Totais & & 42 & & 100 & 100 \\
\hline
\end{tabular}

trado nos trabalhos de Caram (2007), Carvalho et al. (2012), Wanderley et al. (2009) e Wanderley et al. (2012). A covariância calculada nas quatro direções horizontais não mostrou diferenças significativas, desta forma o semivariograma foi considerado isotrópico. O tamanho do passo utilizado foi de $7.100 \mathrm{~m}$, e o número de passos foi de 6 .

No semivariograma anual selecionado foi utilizada a transformação log para uma melhor aderência da distribuição normal aos dados, à covariância negativa apareceu aos $20 \mathrm{~km}$, o alcance (a) foi de $42,6 \mathrm{~km}$, o que delimita o grau de homogeneização da amostra, o patamar $\left(\mathrm{C}_{\mathrm{O}}+\mathrm{C}\right)$ foi de $(0,0329)$, ou seja, a variância se estabilizou neste valor e, por fim, o efeito pepita $\left(\mathrm{C}_{0}\right)$ foi de 0,0006 demonstrando haver irregularidade a curtos espaçamentos (Fig. 6). Ainda assim o valor representou apenas 1,8\% do patamar, valor expressivamente inferior a $30 \%$, limite considerado elevado segundo Andriotti (2003). O IDE, idêntico à porcentagem da relação entre efeito pepita e patamar, foi de $1,82 \%$ representando forte dependência espacial.

Para os dados mensais a covariância negativa apareceu aos $25 \mathrm{~km}$, o alcance foi de $42,6 \mathrm{~km}, \mathrm{o}\left(\mathrm{C}_{\mathrm{O}}+\mathrm{C}\right)$ foi de 965,5487 e o $\left(\mathrm{C}_{\mathrm{O}}\right)$ teve o valor de $(228,1849)$, representando $23,6 \%$ do patamar e forte dependência espacial (Fig. 6).

O modelo teórico gaussiano de semivariograma apresentou os melhores resultados na interpolação anual e mensal por cokrigagem, as covariâncias e o alcance anual e
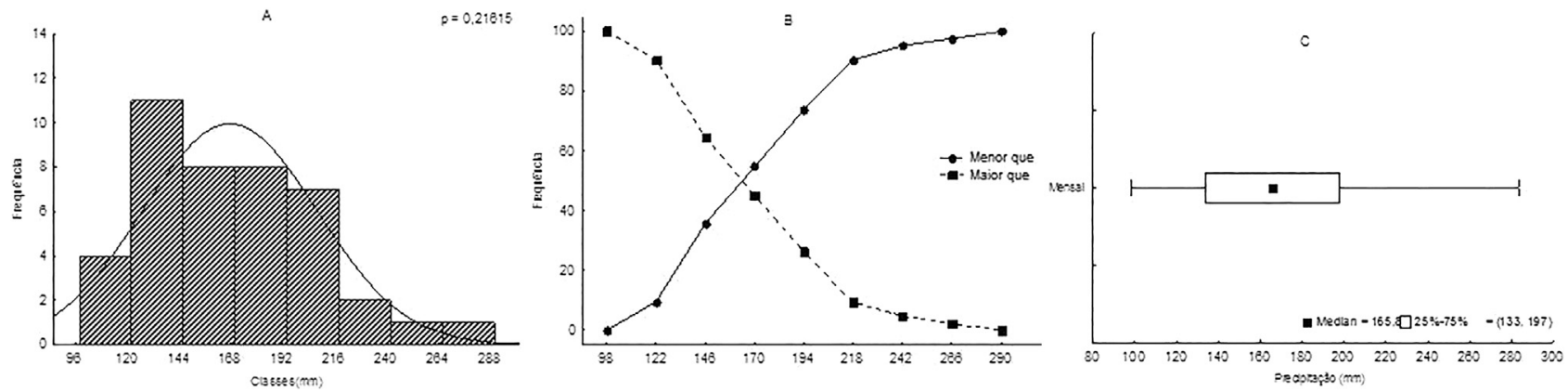

Figura 4 - Gráficos de distribuição dos dados mensais: histograma de frequência (A); ogiva, indicando os eventos menores ou iguais a determinada frequência na forma ascendente e os eventos maiores ou iguais a determinada frequência na forma descendente (B) e boxplot (C). 

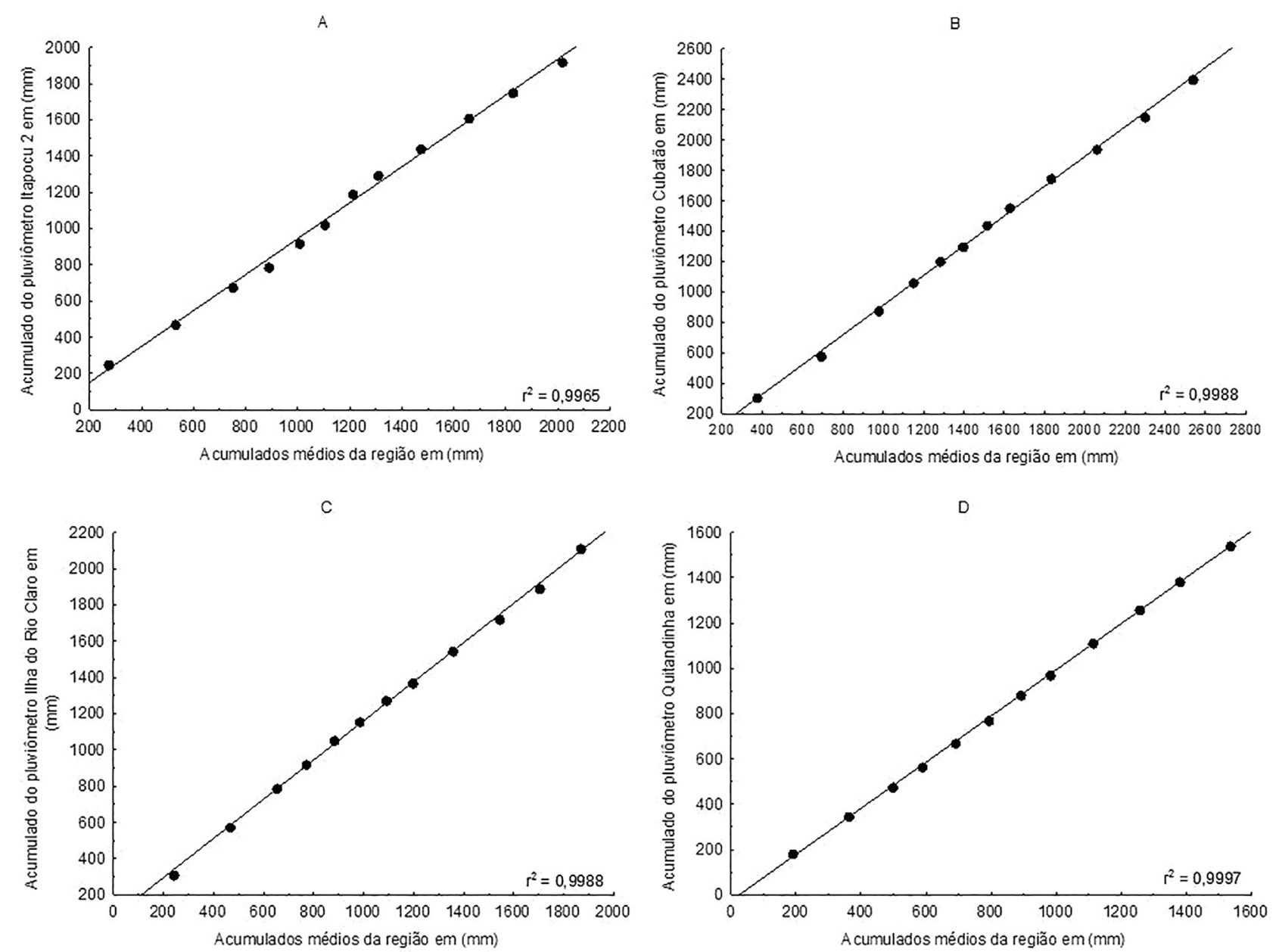

Figura 5 - Regressões lineares dos dados mensais acumulados de precipitação por localidade geográfica dos pluviômetros da planície costeira (A); proximidades da frente da escarpa da Serra do Mar (B); escarpa da Serra do Mar (C) e planalto (D).

mensal foram idênticos aos encontrados utilizando a krigagem. O efeito pepita para os dados anuais de precipitação representou $14,2 \%$ do patamar e para altitude $31,2 \%$, havendo forte e moderada dependência espacial respectivamente. O efeito pepita para os dados mensais de precipitação representou $58,9 \%$ do patamar e para altitude $31 \%$, havendo moderada dependência espacial em ambos os casos.

\subsection{Superfície interpolada pelos métodos de krigagem e cokrigagem}

Através da análise exploratória dos dados disponível na extensão geostatistical analyst optou-se por excluir da amostra o pluviômetro "Estrada dos Morros" com média anual de 3.204, $4 \mathrm{~mm}$, pois esta localizado a uma distância aproximada de $1 \mathrm{~km}$ do posto "Estação Água Azul", que possui média anual de $2.366,6 \mathrm{~mm}$. O pluviômetro excluído configurou-se como outlier nas ferramentas histograma e normal qqplot, além do gráfico de boxplot (Fig. 2-C).

O modelo teórico esférico de semivariograma foi selecionado através da técnica de validação cruzada que utiliza para comparar os modelos às estatísticas de erro médio (ME), que deve ser próximo a (0), raiz quadrada do erro médio padronizado (RMSSE), que deve ser próximo a (1), erro padrão médio (ASE) e raiz quadrada do erro médio (RMSE) que devem ter os valores próximos e menores possível. A superfície anual interpolada apresentou valores de erro médio $(-0,01)$ e raiz quadrada do erro médio padronizado (1,09). O erro padrão médio foi de $214,68 \mathrm{~mm}$ e a raiz quadrada do erro médio de $215,36 \mathrm{~mm}$, ou seja, a previsão foi subestimada em $(0,68 \mathrm{~mm})$.

O mapa de precipitação anual para o município de Joinville (Fig. 7 - A) elaborado pelo método de krigagem ordinária apresentou o valor mínimo de $1.604,3$ mm, máximo de $2.594,2 \mathrm{~mm}$, média de $2.130,1 \mathrm{~mm}$ e desvio padrão de $238,7 \mathrm{~mm}$.

A superfície de erros (Fig. 7 - B) para os dados anuais mostrou que a previsão em relação ao valor medido variou entre $(-588,9 \mathrm{~mm})$ e $519,3 \mathrm{~mm}$, sendo que a faixa predominante de erros esta compreendida entre $(-50 \mathrm{~mm})$ e $50 \mathrm{~mm}$. Os valores negativos indicam que a previsão foi inferior ao valor medido e vice-versa. Estes valores se justi- 

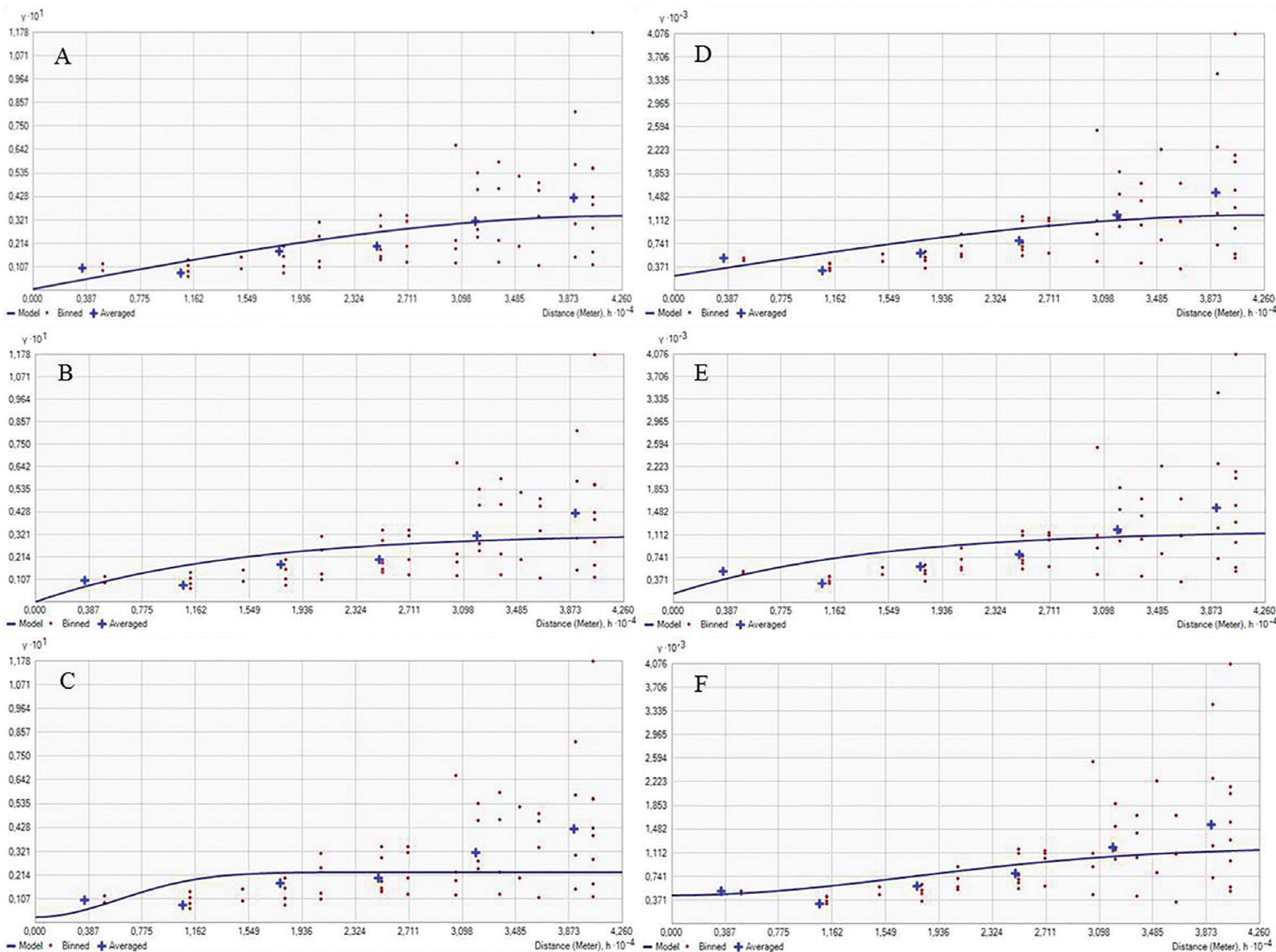

Figura 6 - Semivariogramas empíricos e teóricos ajustados ao conjunto de dados. Anuais: a) esférico; b) exponencial; c) gaussiano. Mensais: d) esférico; e) exponencial; f) gaussiano.
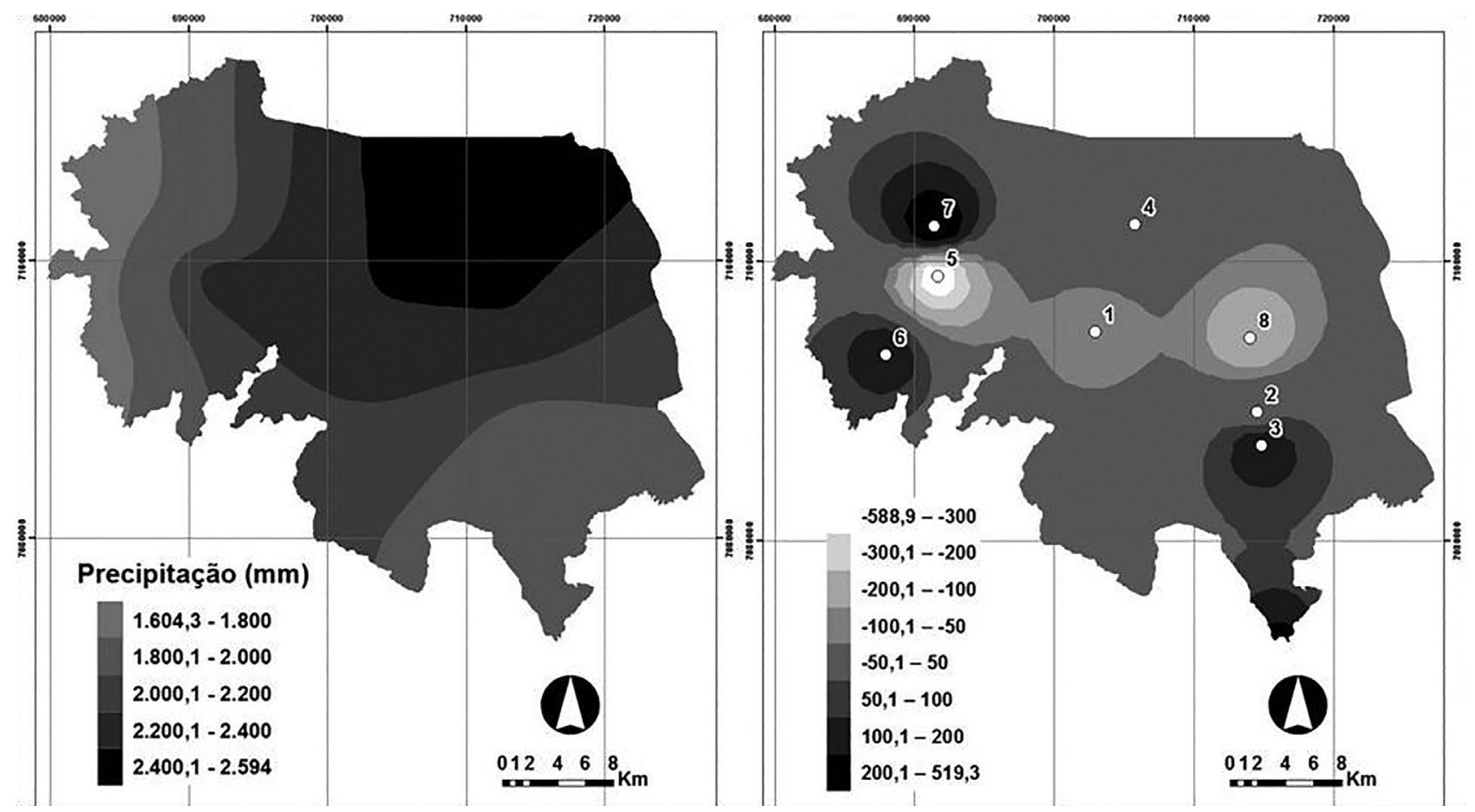

Figura 7 - Superfície interpolada pelo método de krigagem ordinária de precipitação anual para o município de Joinville (A). Superfície representando os erros de estimação de precipitação anual pelo método inverso do quadrado da distância para Joinville (B). 


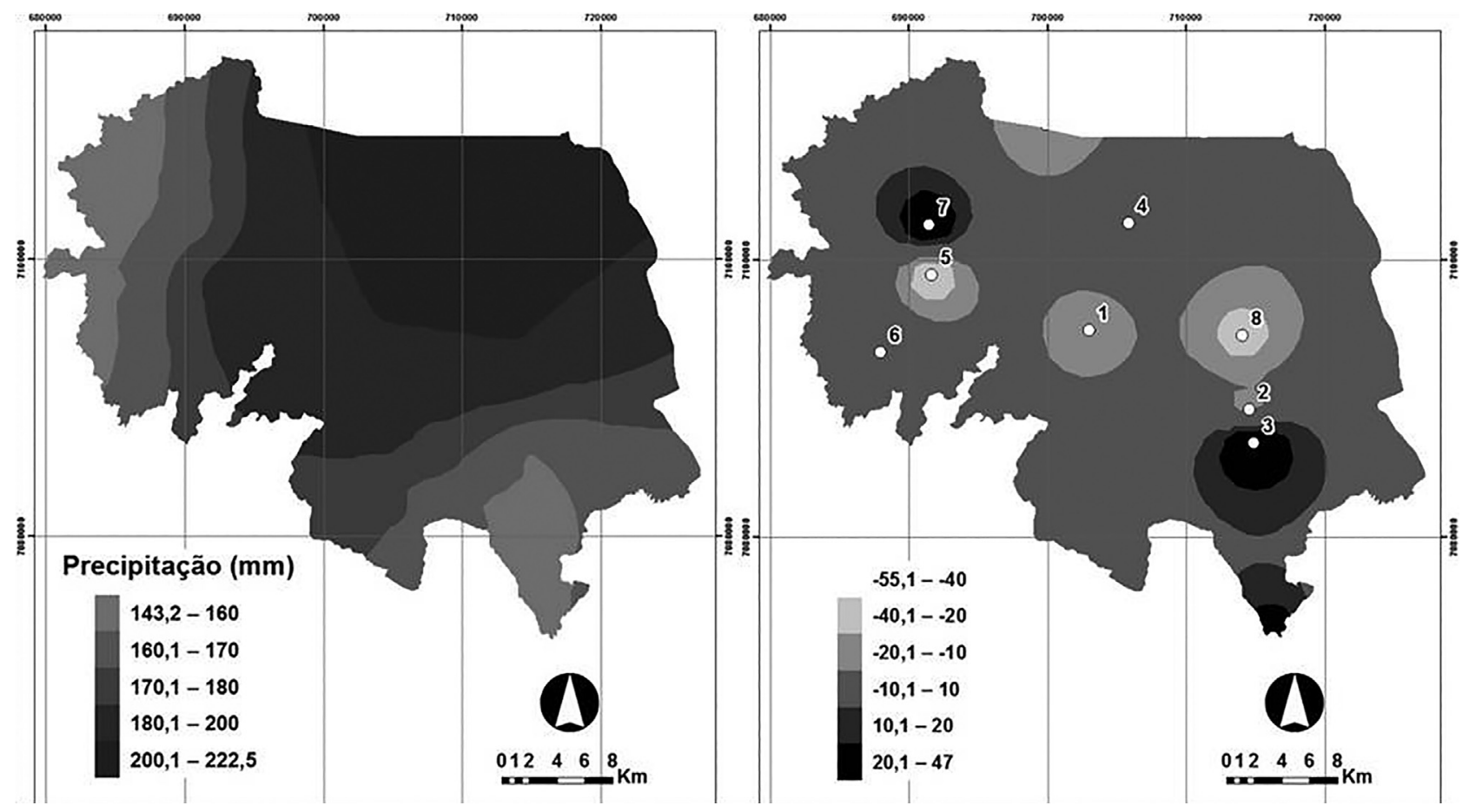

Figura 8 - Superfície interpolada pelo método de krigagem ordinária de precipitação mensal para o município de Joinville (A). Superfície representando os erros de estimação de pelo método inverso do quadrado da distância para Joinville (B).

ficam porque o método interpolador tem a tendência de suavizar a previsão de acordo com os elementos próximos, neste caso o mapa de erros destaca os pluviômetros que não são tão homogêneos em relação à vizinhança.

O mapa de precipitação mensal para Joinville (Fig. 8 - A) apresentou o valor mínimo de $143,2 \mathrm{~mm}$, máximo de 222,5 mm, média de 183,6 mm e desvio padrão de $18,7 \mathrm{~mm}$. A superfície interpolada apresentou valores de erro médio $(0,01)$ e raiz quadrada do erro médio padronizado $(0,93)$. $O$ erro padrão médio foi de $25,25 \mathrm{~mm}$ e a raiz quadrada do erro médio $22,49 \mathrm{~mm}$, ou seja, a previsão foi superestimada em $2,76 \mathrm{~mm}$.

A superfície de erros (Fig. 8 - B) para os dados mensais indicou que os valores previstos em relação aos medidos variaram entre $(-55,1 \mathrm{~mm})$ a $47 \mathrm{~mm}$, sendo a faixa predominante entre $(-10 \mathrm{~mm})$ e $10 \mathrm{~mm}$. Os pluviômetros nomeados como 3 e 7 pelo ArcMap apresentaram elevados erros nas superfícies de erros anuais e mensais, porém o pluviômetro 6 apresentou apenas média anual discrepante em relação a vizinhança.

O mapeamento por cokrigagem exige que a correlação entre a variável primária e secundária seja alta (Yamamoto et al., 2013). Para os dados de precipitação anual e mensal em relação à altitude a correlação foi classificada como positiva moderada, apresentando coeficiente de correlação de $(0,41)$ e $(0,44)$ respectivamente. $O$ gráfico de dispersão demonstrou que os dados de chuva nas maiores altitudes são menores e a variação das médias anuais e mensais abaixo de 300 metros foi alta.
Os valores de precipitação anual e mensal mínimo, máximo, média e desvio padrão ficaram próximos aos encontrados na interpolação por krigagem. Para os dados anuais a variação dos valores foi de $0,2 \%$ a $7,8 \%$, sendo a média de $1.617,5 \mathrm{~mm}$. Os erros ficaram próximos aos encontrados na interpolação por krigagem, o ME foi de $(0,008)$ inferior, a previsão média foi subestimada em $25,86 \mathrm{~mm}$, se destacando como um ponto positivo a favor da krigagem que registrou apenas $0,68 \mathrm{~mm}$ de subestimação.

A diferença dos valores mínimo, máximo, média e desvio padrão, entre os dados mensais variaram entre 1,1\% e 5,3\%, sendo a média de 181,6 mm. A RMSE foi de 22,11 $\mathrm{mm}$ e o ASE de $23,47 \mathrm{~mm}$, significando uma previsão média superestimada em apenas $(1,36 \mathrm{~mm})$, este resultado foi mais significante do que os encontrados por krigagem. Na Fig. 9 é possível observar a distribuição média das chuvas anuais e mensais resultante da interpolação por cokrigagem.

A análise da distribuição de chuvas no município de Joinville foi similar a encontrada em Mello et al. (2013), os maiores valores anuais e mensais estão concentrados mais ao norte do município, próximo a Garuva, região da serra do Quiriri e zona rural de Pirabeiraba, os menores índices pluviométricos distribuem-se no planalto e zona sul de Joinville. As superfícies interpoladas por cokrigagem tiveram valores de precipitação altos distribuídos mais amplamente no perímetro municipal, as isoietas se estenderam no sentido sul. 


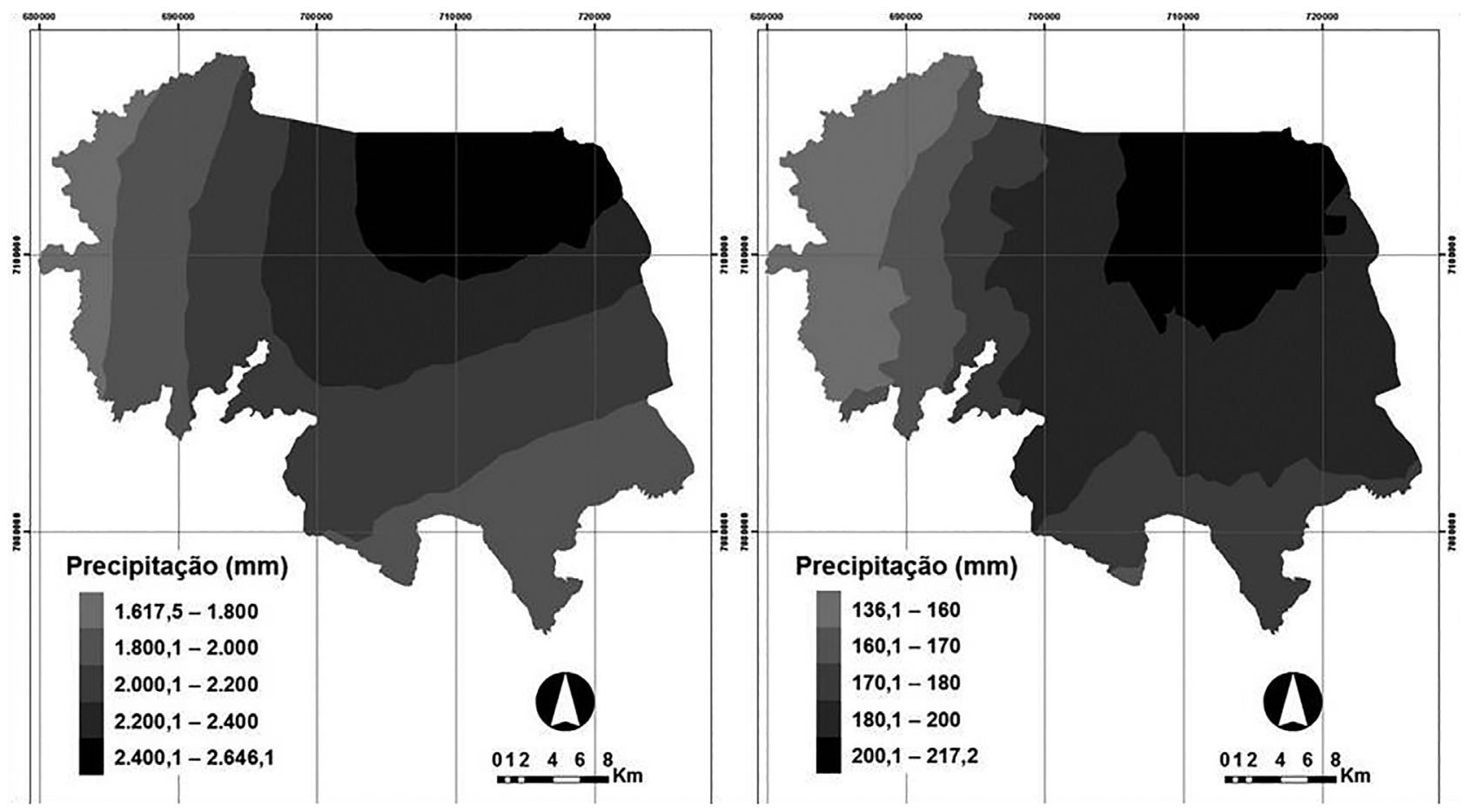

Figura 9 - Superfície interpolada pelo método de cokrigagem ordinária de precipitação anual para o município de Joinville (A). Superfície interpolada pelo método de cokrigagem ordinária de precipitação mensal para o município de Joinville (B).

\section{Conclusões}

A análise exploratória e de homogeneidade contribuiu para o conhecimento prévio da distribuição dos dados, verificando a possibilidade de utilização da amostra e a exclusão do posto outlier "Estrada dos Morros".

A geoestatística permitiu aprofundar a análise espacial dos dados, o IDE foi forte na interpolação por krigagem e na cokrigagem anual para a precipitação, moderado na cokrigagem anual para a altitude e na mensal para altitude e precipitação. Os erros encontrados na comparação dos métodos por validação cruzada foram similares entre a krigagem e a cokrigagem, porém a cokrigagem foi inferior na análise de dependência espacial dos dados, além de apresentar moderada correlação.

Sendo assim as médias de precipitação anual $2.130,1 \mathrm{~mm}$ e mensal $183,6 \mathrm{~mm}$ para Joinville foram selecionadas a partir da krigagem, os maiores volumes médios de chuva em ambos os casos espacializaram-se próximos a serra do Quiriri, mais ao norte do município, influenciando a região de Pirabeiraba.

\section{Referências}

ALVARES, C.A. Mapeamento e modelagem edafoclimática da produtividade de plantações de Eucalyptus no sul do Estado de São Paulo. Tese (Doutorado em Ciências) Escola Superior de Agricultura "Luiz de Queiroz"/ Universidade de São Paulo, 2011.
ANDRIOTTI, J.L.S. Fundamentos de estatística e geoestatística. São Leopoldo: Editora Unisinos/ Universidade do Vale do rio dos Sinos, 2003.

ASSIS, F.N. de. Aplicações de estatística a climatologia: teoria e prática. Francisco N. de Assis, Hermano V. de Arruda, Antonio R. Pereira. - Pelotas: Ed. Universitária/UFPel, 1996.

BARGAOUI, Z.K.; CHEBBI, A. Comparison of two kriging interpolation methods applied to spatiotemporal rainfall. Journal of Hidrology, v. 365, p. 56-73, 2009.

BAÚ, A.L.; GOMES, B.M.; QUEIROZ, M.M.F. de.; OPAZO, M.A.U.; SAMPAIO, S.C. Comportamento espacial da precipitação pluvial mensal provável da mesorregião oeste do Estado do Paraná. Irriga, Botucatu, v. 11, n .2, p. 150-168, abril-junho, 2006.

CARAM, R. de O. Reconstrução de séries e análise geoestatística da precipitação no Estado de Minas Gerais. Dissertação (Magister Scientiae) - Universidade Federal de Viçosa, 2007.

CARVALHO, J.R.P. de; ASSAD, E.D. Análise espacial da precipitação pluviométrica no Estado de São Paulo: comparação de métodos de interpolação. Engenharia Agrícola, v. 25 , p. 377-384, 2005.

CARVALHO, J.R.P. de; ASSAD, E.D.; PINTO, H.S. Interpoladores geoestatísticos na análise da distribuição espacial da precipitação anual e de sua relação com altitude. Pesquisa Agropecuária Brasileira, v. 47, n. 9, p. 1235-1242, set. 2012.

CARVALHO, J.R.P. de; QUEIROZ, E.F. de. Uso de cokrigagem colocalizada na determinação da distribuição espacial de precipitação. Campinas: Embrapa Informática Agropecuária, Comunicado Técnico, 4 p., 2002. 
CARVALHO, J.R.P. de; VIEIRA, S.R. Validação de modelos geoestatísticos usando teste de Filliben: aplicação em agroclimatologia. Campinas: Embrapa Informática Agropecuária. Comunicado Técnico, 4 p., 2004.

DIODATO, N. The influence of topography co-variables on the spatial variability of precipitation over small regions of complex terrain. International Journal of Climatology, v. 25 , p. 351-363, 2005.

HEVESI, J.A.; FLINT, A.L.; ISTOK, J.D. Precipitation estimation in mountainous terrain using multivariate geostatistics. Part II: isohyetal maps. Journal of applied meteorology, v. 31, p. 677-688, 1992.

IPPUJ - Fundação Instituto de Pesquisa e Planejamento para o Desenvolvimento Sustentável de Joinville (Org.). Joinville cidade em dados 2013. Joinville: Prefeitura Municipal, 2013, $207 \mathrm{p}$.

LÉLLIS, F.S.; BARROSO, G.F. Modelagem geoestatística de precipitação pluviométrica na bacia do rio Jacaraípe, Serra (ES). Anais XV Simpósio Brasileiro de Sensoriamento Remoto, Curitiba, PR, Brasil, INPE, p.5201, 2011.

MELLO, C.R.; LIMA, J.M.; SILVA, A.M.; MELLO, J.M.; OLIVEIRA, M.S. Krigagem e inverso do quadrado da distância para interpolação dos parâmetros da equação de chuvas intensas. R. Bras. Ci. Solo, v. 27, p. 925-933, 2003.

MELLO, J.M. de. Geoestatística aplicada ao inventário florestal. Tese (Doutor em Recursos Florestais) - Escola Superior de Agricultura "Luiz de Queiroz"/ Universidade de São Paulo, 2004.

MELLO, J.M. de.; BATISTA, J.L.F.; JÚNIOR, P.J.R.; OLIVEIRA, M.S. de. Ajuste e seleção de modelos espaciais de semivariograma visando a estimativa volumétrica de Eucalyptus grandis. Scientia Forestalis, n. 69, p. 25-37, dez. 2005.

MELLO, Y.R. de; KOEHNTOPP, P.I.; OLIVEIRA T.M.; VAZ, C.. Distribuição de precipitação pluviométrica na região de Joinville. Trabalho de Conclusão de Curso (Graduação em Geografia). Universidade da Região de Joinville - Univille. Joinville, 2013.
MOULIN, G.D.. Técnicas de entropia e de geoestatística no dimensionamento de redes pluviométricas. Dissertação (Mestre em Engenharia Ambiental) - Universidade Federal do Espírito Santo, 2005.

OLIVEIRA, F.A. de. Estudo do aporte sedimentar em suspensão na baía da babitonga sob a ótica da geomorfologia. Tese (Doutor em Geografia) - Universidade de São Paulo - USP. Faculdade de Filosofia, Letras e Ciências Humanas, 2006.

RODRIGUES, M.L.G.; FRANCO, D.; SUGAHARA, S. Climatologia de frentes frias no litoral de Santa Catarina. Revista Brasileira de Geofísica, p. 135-151, 2004.

TRIOLA, M.F. Introdução a estatística/ Mario F. Triola; tradução Vera Regina Lima de Farias e Flores, revisão técnica Ana Maria Lima de Farias. 10. ed. Rio de Janeiro: LTC, 2008.

TUCCI, C.E.M. Hidrologia: ciência e aplicação. 2. ed.; 2. reimpr. - Porto Alegre: Ed. Universidade/ UFRGS: ABRH, 2001.

VIEIRA, S. Introdução a bioestatística/ Sonia Vieira. - 3. ed. revista e ampliada. - Rio de Janeiro: Campus, 1980.

VIOLA, M.R.; MELLO, C.R. de; PINTO, D.B.F.; MELLO, J.M. de; ÁVILA, L.F. Métodos de interpolação espacial para o mapeamento da precipitação pluvial. Revista Brasileira de Engenharia Agrícola e Ambiental, v. 14, n. 9, p. 970-978, 2010.

WANDERLEY, H.S.; AMORIM, R.F.C.; CARVALHO, F.O. de. Variabilidade espacial e preenchimento de falhas de dados pluviométricos para o Estado de Alagoas. Revista Brasileira de Meteorologia, v. 27, n. 3, p. 347-354, 2012.

WANDERLEY, H.S.; CARVALHO, F.O. de; AMORIM, R.F.C.; CAVALCANTI, J.R. de A.; SANTOS, R.R.C. Análise geoestatística da precipitação para o Estado de Alagoas. XVII Simpósio Brasileiro de Recursos Hídricos. Campo Grande/MS, 2009.

YAMAMOTO, J.K. Geoestatística: conceitos e aplicações. Jorge Kazuo Yamamoto, Paulo M. Barbosa Landim. São Paulo: Oficina de Textos, 2013.

All the contents of this journal, except where otherwise noted, is licensed under a Creative Commons Attribution License CC-BY. 